\title{
THE PICARD THEOREM FOR RIEMANN SURFACES
}

\author{
H. L. ROYDEN
}

\begin{abstract}
Let $W$ be a Riemann surface other than the sphere, plane, punctured plane or torus. Let $f$ be a holomorphic map of the punctured disk $0<|z|<1$ into $W$. Then $f$ can be extended to a holomorphic map of the disk $|z|<1$, possibly, into a Riemann surface $W^{*}$ containing $W$. We give a new proof of this fact and explore some consequences of it.
\end{abstract}

Every Riemann surface, except the sphere, torus, plane and punctured plane, has the unit disk as its universal covering surface. Let us call any Riemann surface other than these four nonexceptional. The following theorem is a generalization of the "big" Picard Theorem for mappings of the punctured disk into one of the nonexceptional Riemann surfaces.

THEOREM. Let $\varphi$ be a holomorphic map of the punctured disk $\Delta_{0}=\{z$ : $0<|z|<1\}$ into a nonexceptional Riemann surface $W$. Then either $\varphi$ extends to a holomorphic map of the disk $\Delta=\{z:|z|<1\}$ into $W$ or else $W$ is contained in a Riemann surface $W^{*}=W \cup\{p\}$, so that $\varphi$ extends to a holomorphic map of the disk $\Delta$ into $W^{*}$.

This beautiful theorem is implicit in the work of Ohtsuka of three decades ago. Ohtsuka [4] and Huber [2] show that if $\varphi$ is a holomorphic map of the punctured disk into a nonexceptional Riemann surface $W$, and if $\varphi$ does not extend to a map of the disk into $W$, then $\varphi(z)$ clusters at a single end of $W$ as $z \rightarrow 0$. Ohtsuka observes [5] that such an end must be planar. The theorem follows easily from these two facts. The theorem seems, unfortunately, to be almost unknown, probably because Ohtsuka's statements and proofs are formulated in terms of the larger framework of cluster set theory for maps into an open Riemann surface. The purpose of this note is to give a direct proof based on the properties of covering surfaces and to derive several useful corollaries.

Heins [1] and Marden, Richards and Rodin [3] have given simplified proofs. These follow Ohtsuka in first showing that the values of $\varphi$ can only cluster at a single end of $W$ as $z \rightarrow 0$ and then using topological arguments on $W$ to show that this end is planar.

We proceed by considering $W$ as the quotient $U / \Gamma$ of the upper half-plane $U$ by a discrete group $\Gamma$ of conformal self-maps of $U$. Each element of $\Gamma$ (other than the identity) is a linear fractional transformation without fixed points in $U$ and having

Received by the editors September 20, 1982 and, in revised form, June 22, 1983.

1980 Mathematics Subject Classification. Primary 30F99.

(C1984 American Mathematical Society $0002-9939 / 84 \$ 1.00+\$ .25$ per page 
either one or two fixed points on the boundary of $U$. The former are called parabolic elements, the latter hyperbolic. An element $T \in \Gamma$ is said to be primitive if $T$ is not $S^{n}$ for some $S \in \Gamma$ and $n>1$. If $\Gamma_{1}$ is a subgroup of $\Gamma$ generated by an element $T$, then $U / \Gamma_{1}$ is conformally equivalent to a ring domain $R=\{z: a<|z|<1\}$. The generator $T$ is parabolic if and only if $a=0$, i.e., iff $R=\Delta_{0}$.

Let $T$ be a primitive parabolic element of $\Gamma$ and let $\Gamma_{1}$ be the subgroup generated by $T$. Changing coordinates in $U$ by a conformal self-map so that the fixed point of $T$ is at $\infty$, we may take $T$ to be the translation $T(\zeta)=\zeta+1$. The function $e^{2 \pi i \zeta}$ maps $U / \Gamma_{1}$ one-to-one onto $\Delta_{0}$. Let $\hat{\psi}: \Delta_{0} \rightarrow U / \Gamma_{1}$ be the inverse of this mapping, and set $\psi=\pi \circ \hat{\psi}$, where $\pi$ is the projection of $U / \Gamma_{1}$ onto $U / \Gamma=W$. Thus $\psi$ maps $\Delta_{0}$ onto $W$. Moreover, $\psi$ is a covering map since $\pi$ and $\hat{\psi}$ are.

Lemma 1. The map $\psi: \Delta_{0} \rightarrow W$ is univalent on the punctured disk $\left\{z: 0<|z|<e^{-\pi}\right\}$.

Proof. The lemma is equivalent to the statement that no two points in the half-strip $\left\{\zeta: 0 \leqslant \operatorname{Re} \zeta<1, \operatorname{Im} \zeta>\frac{1}{2}\right\}$ are identified by an element $S$ of $\Gamma$. Since $T$ is primitive, no translation in $\Gamma$ can identify any two points in the $\operatorname{strip}\{\zeta: 0 \leqslant \operatorname{Re} \zeta<$ 1). Let $S$ be an element of $\Gamma$ which is not a translation. Then

$$
S(\zeta)=(a \zeta+b) /(c \zeta+d)
$$

with $a, b, c, d$ real, $a d-b c=1$ and $c \neq 0$. The fact that $S$ has no fixed point in the upper half-plane implies $|a+d| \geqslant 2$. Since

$$
S T^{n}=(a \zeta+a n+b) /(c \zeta+c n+d)
$$

is also an element of $\Gamma$, we have $|a+d+c n| \geqslant 2$ for each integer $n$. We conclude from this and $c \neq 0$ that $|c| \geqslant 2$. For $\zeta=\xi+i \eta$ we have

$$
\operatorname{Im} S(\zeta)=\frac{(a d-b c) \eta}{(c \xi+d)^{2}+c^{2} \eta^{2}} \leqslant \frac{1}{c^{2} \eta}<\frac{1}{2},
$$

provided $\eta>\frac{1}{2}$. Thus $\operatorname{Im} \zeta>\frac{1}{2}$ implies $\operatorname{Im} S(\zeta)<\frac{1}{2}$, so $S$ cannot identify distinct points of $\left\{\zeta: \operatorname{Im} \zeta>\frac{1}{2}\right\}$.

LEMma 2. There is a Riemann surface $W^{*}=W \cup\{p\}$ such that the map $\psi$ has a holomorphic extension to a map $\psi^{*}: \Delta \rightarrow W^{*}$.

Proof. Let $C$ be the image under $\psi$ of the circle $|z|=e^{-2 \pi}$ and let $D$ be the image of $\left\{z: 0<|z|<e^{-2 \pi}\right\}$. Since $\psi$ is univalent on $\left\{z: 0<|z|<e^{-\pi}\right\}, C$ is a simple closed analytic curve and $D$ is (conformally equivalent to) a punctured disk. If we know that $C$ separates $D$ from $W \sim(C \cup D)$ in $W$, we can replace $D$ by a disk $D^{*}=D \cup\{p\}$ so that $W^{*}=W \cup\{p\}$ is a Riemann surface. The restriction of the map $\psi$ to $\left\{z: 0<|z|<e^{-2 \pi}\right\}$ extends to a holomorphic map of $\left\{z:|z|<e^{-2 \pi}\right\}$ into $D^{*}$ by the Riemann removable discontinuity theorem, and, hence, $\psi$ has a holomorphic extension $\psi^{*}: \Delta \rightarrow W^{*}$.

To see that $C$ separates $D$ from $W \sim(C \cup D)$, we take an arc $\gamma$ joining a point $p_{1} \in D$ to a point $p_{2} \in W \sim(C \cup D)$. Now $p_{1}=\psi\left(z_{1}\right)$, where $\left|z_{1}\right|<e^{-2 \pi}$ and $\psi$ is a covering map. Thus $\gamma$ lifts to an arc in $\Delta_{0}$ joining $z_{1}$ to a point $z_{2}$ with $\psi\left(z_{2}\right)=p_{2}$. Since $\left|z_{2}\right|>e^{-2 \pi}$, the lifted arc meets the circle $|z|=e^{-2 \pi}$, so $\gamma$ must meet $C$. 
The reader should note that, if an annulus (or equivalently a punctured disk) $D$ together with its outer boundary $C$ are topologically embedded in a surface $W$, then $D$ can be replaced by a disk to get a (Hausdorff) surface if and only if $C$ separates $D$ from $W \sim(C \cup D)$.

These two lemmas assert in effect that a parabolic element $T$ in $\Gamma$ arises because the surface $W=U / \Gamma$ is obtained by puncturing a surface $W^{*}$ with $T$ corresponding to a class of curves homotopic to the puncture. This fact is classical when $\Gamma$ is finitely generated.

LEMMA 3. Let $\varphi$ be a holomorphic map of $\Delta_{0}$ into a ring domain $R=\{z$ : $a<|z|<1\}$. Then either $\varphi$ extends to a holomorphic map of $\Delta$ into $R$ or else $a=0$ and $\varphi$ extends to a holomorphic map of $\Delta$ into $\Delta=R \cup\{0\}$.

Proof. Since $\varphi$ maps $\Delta$ into $R \subset \Delta, \varphi$ is bounded. Hence $\varphi$ extends to a map of $\Delta$ into $\Delta$ by the Riemann removable discontinuity theorem. Since the image of $\Delta$ is an open set contained in $R \cup\{\varphi(0)\}$, we must have $\varphi(0) \in R$ or else $\varphi(0)=0$ and $a=0$.

Proof of Ohtsuka's Theorem. We consider $W$ to be $U / \Gamma$ and $\Delta_{0}=U / G$, where $G$ is the group of self-maps of $U$ generated by $T_{1}(\zeta)=\zeta+1$. Then the map $\varphi$ lifts to a map $\hat{\varphi}: U \rightarrow U$, and $\hat{\varphi}$ induces a homomorphism $h: G \rightarrow \Gamma$ so that $\hat{\varphi} \circ S=h(S) \circ \hat{\varphi}$. If $\varphi$ takes $|z|=\frac{1}{2}$ into a curve homotopic to zero, we have $h[G]=\{I\}$, and the map $\varphi$ lifts to a map $\tilde{\varphi}: \Delta_{0} \rightarrow U$. Since $\operatorname{Re} \tilde{\varphi}>0$ in $\Delta_{0}, \tilde{\varphi}$ extends to a map $\tilde{\varphi}^{*}$ of $\Delta$ into $U$. If $\pi$ is the projection of $U$ onto $U / \Gamma=W$, then $\varphi^{*}=\pi \circ \tilde{\varphi}^{*}$ is a holomorphic extension of $\varphi$ which maps $\Delta$ into $W$.

If, on the other hand, $h\left(T_{1}\right) \neq I$, then $h\left(T_{1}\right)=T^{n}$ for some primitive element $T$ of $\Gamma$. Let $\Gamma_{1}$ be the subgroup generated by $T$. Since $h[G] \subset \Gamma_{1}$, the map $\varphi$ lifts to a map $\tilde{\varphi}$ of $\Delta_{0}$ into $R=U / \Gamma_{1}$. Lemma 3 implies $R=\Delta_{0}$, whence $T$ is parabolic. Moreover, $\tilde{\varphi}$ extends to a holomorphic map $\tilde{\varphi}^{*}$ of $\Delta$ into $\Delta$. We have $\varphi=\psi \circ \tilde{\varphi}$, and Lemma 2 states that $\psi$ extends to a holomorphic map $\psi^{*}$ of $\Delta$ into $W^{*}$. Thus $\varphi^{*}=\psi^{*} \circ \tilde{\varphi}^{*}$ is the desired holomorphic extension of $\varphi$.

If $\varphi$ is a map of $\Delta_{0}$ into a nonexceptional Riemann surface $W$, and if $\varphi$ does not extend to a holomorphic map of $\Delta$ into $W$, then its extension $\varphi^{*}$ must take 0 into $p$, which was not in $W$. For such a map and any sequence $z_{n} \rightarrow 0$, we must have $\varphi\left(z_{n}\right) \rightarrow p$ in $W^{*}$, so $\varphi\left(z_{n}\right)$ has no cluster point in $W$. Thus we have:

COROllaRY 1. Let $\varphi$ be a holomorphic map of $\Delta_{0}$ into a nonexceptional Riemann surface $W$ and suppose, for some sequence $z_{n} \rightarrow 0$, the image sequence $\varphi\left(z_{n}\right)$ has a cluster point in $W$. Then $\varphi$ extends to a holomorphic map of $\Delta$ into $W$.

COROLlARY 2. Let $W$ be a nonexceptional Riemann surface which is contained in a Riemann surface $W_{1}$ and suppose the closure $\bar{W}$ of $W$ in $W_{1}$ is compact. Then each holomorphic map $\varphi$ of $\Delta_{0}$ into $W$ extends to a holomorphic map of $\Delta$ into $W_{1}$.

Proof. Let $p_{1}, p_{2}$ and $p_{3}$ be three distinct points of $W$. If one of these is a cluster point of $\varphi\left(z_{n}\right)$ for some sequence $z_{n} \rightarrow 0$, then $\varphi$ extends to a holomorphic map of $\Delta$ into $W$ by Corollary 1. If not, there is an $a>0$ such that $\varphi$ maps $\Delta_{0}^{a}=\{z$ : $0<|z|<a\}$ into $W \sim\left\{p_{1}, p_{2}, p_{2}\right\} \subset W_{2}=W_{1} \sim\left\{p_{1}, p_{2}, p_{3}\right\}$. Since $\bar{W}$ is compact, $\varphi(1 / n)$ has a cluster point $p \in \bar{W} \subset W_{1}$. But $p \neq p_{i}$, so $p \in W_{2}$. Since $W_{2}$ is 
nonexceptional, Corollary 1 implies that $\varphi$ restricted to $\Delta_{0}^{a}$ has a holomorphic extension to $W_{2} \subset W_{1}$. Combining this extension with $\varphi$ in $a \leqslant|z|<1$ gives the desired extension mapping $\Delta$ into $W_{1}$.

COROLlary 3. Let $V^{\prime}$ be a Riemann surface obtained by deleting $N$ points from a compact Riemann surface $V$ of genus $G$, and $W^{\prime}$ obtained by deleting $n$ points from $a$ compact Riemann surface of genus $g$. Suppose $2 g+n \geqslant 3$. Then every holomorphic map $\varphi$ of $V^{\prime}$ into $W^{\prime}$ extends to a holomorphic map of $V$ into $W$. If $\varphi$ is nonconstant, then $G \geqslant g$ and $N \geqslant n$.

Proof. Corollary 2 implies that $\varphi$ extends to a holomorphic map of $V$ into $W$. If $\varphi$ is nonconstant, it is onto and gives $V$ as a ramified cover of $W$. Since $\varphi$ is onto, the inverse images of points deleted from $W$ are points deleted from $V$. Hence $N \geqslant n$. If $\varphi$ has order $m \geqslant 1$, the Riemann-Hurwitz formula gives $2(G-1)=2 m(g-1)+\sum r_{i}$ where the $r_{i}$ are the ramification indices at the branch points of $\varphi$. Since these are positive and $m \geqslant 1$, we have $2(G-1) \geqslant 2(g-1)$ if $g>0$. Thus $G \geqslant g$.

\section{BIBLIOGRAPHY}

1. M. Heins, On Fuchsoid groups that contain parabolic transformations, Contributions to Function Theory, Tata Inst., Bombay, 1960.

2. H. Huber, Über analytische Abbildungen Riemannschen Flächen in sich, Comment. Math. Helv. 27 (1953), 1-73.

3. A. Marden, I. Richards and B. Rodin, Analytic self-mappings of Riemann surfaces, J. Analyse Math. 18 (1967), 197-225.

4. M. Ohtsuka, On the behavior of an analytic function about an isolated boundary point, Nagoya Math. J. 4 (1952), 103-108.

5. B Boundary components of abstract Riemann surfaces, Lectures on Functions of a Complex Variable (W. Kaplan, ed.), Ann Arbor, 1955, pp. 303-307.

Department of Mathematics, Stanford University, Stanford, California 94305

School of Mathematics, Institute for Advanced Study, Princeton, New Jersey 08540 